Supporting Information for:

\title{
Effects of Refolding on Large-Scale RNA Structure
}

Elizabeth A. Dethoff and Kevin M. Weeks*

Department of Chemistry, University of North Carolina, Chapel Hill, NC 27599-3290

*correspondence: weeks@unc.edu

Supporting methods, analysis, figures, and list of supporting files. 
Generation of accepted 5'-UTR and 5' capsid-coding DENV2 structure

An "accepted" DENV2 secondary structure was created by combining and unifying structural elements proposed in early homology-based studies and supported by subsequent structural studies of DENV2 RNA fragments, mini-genomes, and full-length genomes. The 5'-UTR and 5' capsid-coding regions include the following structures: stem-loop $A(S L A)^{1,2}$, stem-loop $B(S L B)^{3}$, capsid-coding region hairpin (CHP) ${ }^{4}$, a pseudoknot downstream of the $5^{\prime}$ cyclization sequence $(\mathrm{DCS}-\mathrm{PK})^{5}$, and capsid-coding regions 1,2 , and 3 (CCR1, CCR2, CCR3) ${ }^{6}$ (Fig. S4A). Many of these structures have been recently supported or verified within the context of mini-genomes or full-length genomes ${ }^{5,7-10}$. Small, but notable, variations in base pairs at the ends of helices and near bulges exist among the published DENV2 structures. If there was no clear consensus for a particular region, base pairs were not included. For example, base pairs in the oligo(U) track were not included in the accepted DENV2 structure because they are not proposed consistently in published DENV2 structures. This stringent definition of accepted base pairs will tend to decrease the ppv for comparisons of ex virion and refolded genome structures relative to a less parsimoniously-defined accepted structure.

\section{Comparison of 5'-UTR ex virion and refolded structures to the accepted structure}

Both the ex virion and refolded structures accurately recover every major structural feature in the 5'-UTR and 5' portion of the capsid-coding region (Figs. S4B, S4C), including the DCS-PK pseudoknot downstream of cHP. The ppv values for a given element are smaller than the sensitivity for both the ex virion and refolded RNA models, because the SHAPE-predicted models predict more base pairs than the accepted DENV2 structure (Fig. 4C). The major difference between the two SHAPE-predicted structures and the accepted DENV2 structure is that both SHAPE-predicted structures predict additional base pairs at the base of SLB involving the conserved oligo(U) track located downstream of SLA (Figs. S4B, S4C; red and purple base pairs). However, the oligo(U) track has previously been proposed to be base paired ${ }^{4-6,9}$. In fact, Liu et al. recently proposed that base pairing to the oligo(U) track constitutes a conformation-tunable element which they termed the 5'UAR-flanking stem (UFS) ${ }^{9}$.In certain flaviviruses, UFS is critical for NS5 recruitment and viral RNA synthesis. Of the flaviviruses studied, the UFS in DENV2 appeared to be the most unstable and is likely dynamic. In agreement with these findings, the UFS base pairs in our ex virion and refolded SHAPE-derived models are not highly probable (data not shown), indicating that we have less confidence in these predicted base pairs.

\section{Generation of accepted 3'-UTR DENV2 structure}

An "accepted" DENV2 secondary structure of the 3'-UTR was created by combining structures from both early homology-based studies and later structural studies of DENV2 RNA (Fig. S5A, S5B). The 3'-UTR includes stem loops SL-II and SL-IV, two dumbbell structures (DB1 and DB2), SHP, and 3'SL ${ }^{11-13}$. In some depictions of the 3'-UTR, SL-II and SL-IV are termed SL1 and SLII, respectively, and SL-III and SL-V are often not specifically named. Also, DB1 and DB2 names are often interchanged or termed 5'DB and 3'DB, respectively. Multiple pseudoknots are proposed to exist in the $3^{\prime}-U T R$, including a pseudoknot in each of the dumbbell structures ${ }^{14}$, and pseudoknots in SL-II and SL-IV (Figs. S5A, S5B, green base pairs) ${ }^{13}$. However, it has previously been observed that pk1 is clearly less stable and does not always form in DENV2 ${ }^{15}$ and that pk2 is only partially stable in the Murray Valley encephalitis virus ${ }^{16}$. Recent crystal structures of elements in the Murray Valley encephalitis virus and Zika virus, similar to SL-IV and SL-II, respectively, have revealed highly compact structures with an additional pseudoknot involving nucleotides in the three-way junction ${ }^{16,17}$. Similar pseudoknots may exist in the DENV2 SL-II and SL-IV but are not depicted here. Many of the $3^{\prime}-U T R$ structures proposed in early studies are supported by recent structural studies $^{7,10,15,18}$.

\section{Comparison of 3'-UTR ex virion and refolded structures to the accepted structure} Both the ex virion and refolded structures accurately recover most of the major structural features 
in the 3'-UTR (Figs. S5C, S5D), including SL-II, SL-III, SL-IV, SL-V, DB1, DB2, and part of the 3'SL. The major differences between the SHAPE-predicted models and the accepted DENV2 structure is that the ex virion and refolded RNA models $(i)$ predict a medium-range base-paired helix involving positions 10247-10270 and 10640-10661 that occludes formation of part of the full 3'SL structure and (ii) do not recover all known pseudoknots.

The medium-range base pairs (involving positions 10247-10270 and 10640-10661) in the refolded and ex virion models are predicted to have a lower probability $(30-80 \%)$ than other predicted structures. (The base pairs for the full 3'SL are predicted, but to be less probable, at 10-30\%.) In addition, evidence for the medium-range interaction was observed directly by RING-MaP (RNA interaction groups by mutational profiling) probing ${ }^{19}$. The observed clustering of RINGs in the 3'UTR further suggested that the 3'-UTR forms a complex three-dimensional fold, where the separate structural domains (e.g. SL-II, SL-IV, DB1, DB2) are topologically constrained by the medium-range base pairs. Therefore, it is not clear that the SHAPE-directed prediction of the medium-range interaction is an error. As such, we calculated the sensitivity and ppv of the SHAPE models by omitting the nucleotides involved in this interaction (Fig. 4C, 3'-UTR*). For completeness, we also report a sensitivity and ppv for the models without omitting these regions.

Our analysis requires a pseudoknot to have a length of at least three base pairs, which precluded us from predicting pk1, which is usually depicted in DENV2 as a two-base pair pseudoknot.

Although our folding pipeline did not predict pk1 and pk2, the low SHAPE reactivity of both the SLII and SL-IV apical loops and their respective base pairing partners support formation of both pk1 and pk2. As pk1 and pk2 were not predicted in our models, the base pairing partners of the apical loops were instead predicted to base pair to other nucleotides at the base of the stem-loops, thus elongating the SL-II and SL-IV structures. Similar elongated structures were previously predicted by conservation analysis, and it was suggested that both mutually exclusive structures might form independently ${ }^{18}$. Finally, the SHAPE-directed refolded model predicts both DB pseudoknots (pk3 and pk4), but the ex virion model only predicts $\mathrm{pk} 3$. A previous structural study also found that $\mathrm{pk} 4$ was less stable than $p k 3^{15}$.

In summary, the ex virion and refolded RNA models recover most features of current accepted models. The SHAPE-directed models miss several short pseudoknots and predict a mediumrange helix (positions 10247-10270 and 10640-10661) that is not included among accepted DENV2 models. Intriguingly, the refolded RNA structure is more similar to the accepted structure than is the ex virion model. This greater similarity with the refolded model may reflect that the accepted structures are based on experiment-free in silico modeling and that experimental validation often uses short, in vitro transcript RNAs, which are essentially examples of refolded RNAs. 

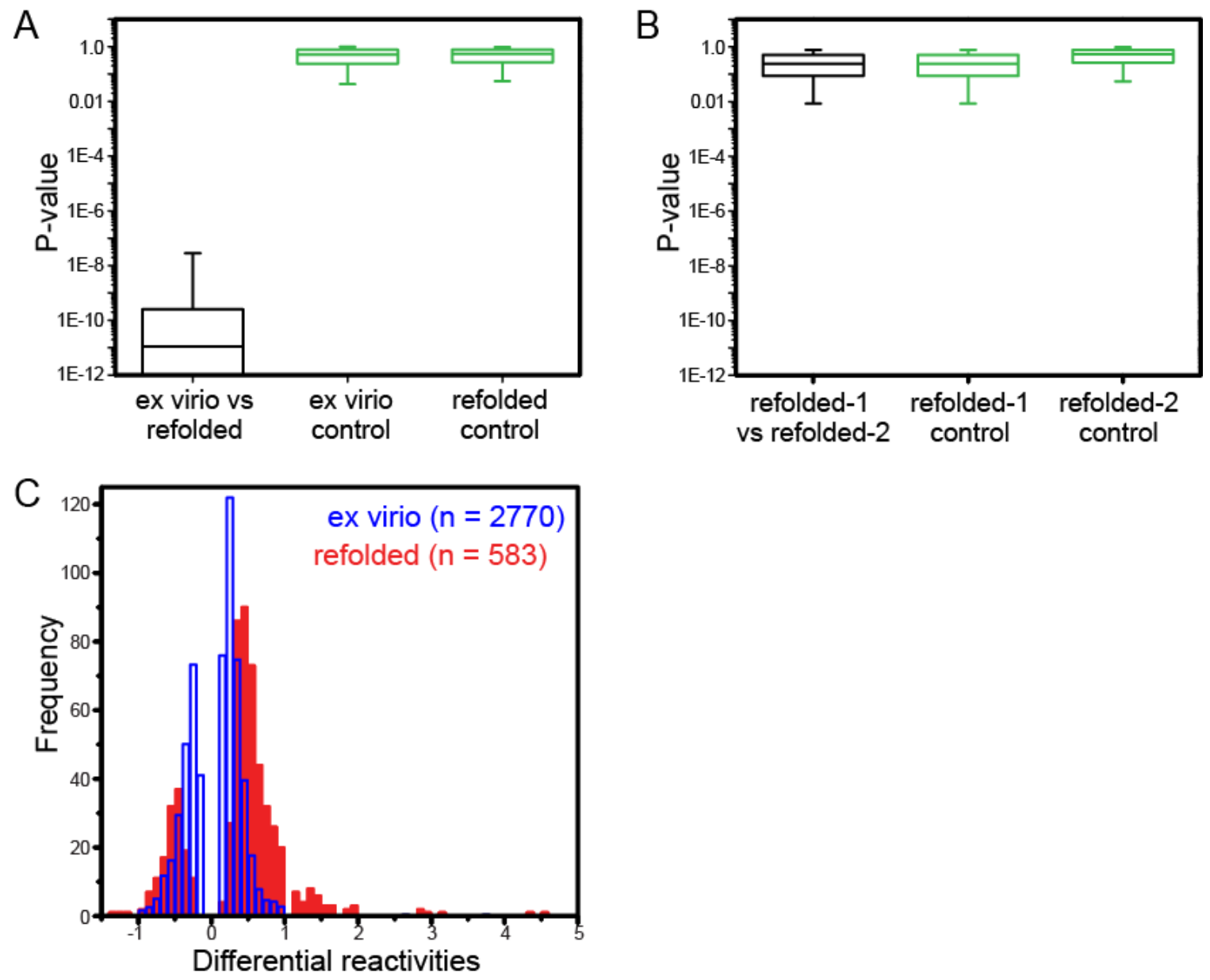

Figure S1: Kolmogorov-Smirnov p-values and differential SHAPE values. (A) Boxplots of the $1000 p$-values from Kolmogorov-Smirnov (KS) permutation tests. The bottom, middle, and top line of each box mark the first, second, and third quartiles, respectively. The whiskers represent the $5^{\text {th }}$ and $95^{\text {th }}$ percentiles. The black boxplot shows the comparison of ex virion and refolded $1 \mathrm{M} 7$ SHAPE reactivities. All $p$ values fell below the Bonferroni-corrected significance threshold value $(\alpha / n=0.00001)$. Control $K S$ tests that compared ex virion and refolded $1 \mathrm{M} 7$ reactivities to themselves are in green. (B) Boxplots of the $1000 p$-values from the refolded RNA replicate KS permutation tests. The black boxplot indicates comparisons of two replicate sets of refolded $1 \mathrm{M} 7$ reactivities. Of the 1000 tests, 999 had $p$ values greater than $\alpha / n$. Two control KS tests that compared refolded RNA replicate 1 and refolded RNA replicate 2 reactivities to themselves are in green. (C) Histograms of significant differential SHAPE reactivities in ex virion (blue) and refolded (red) RNA states. 


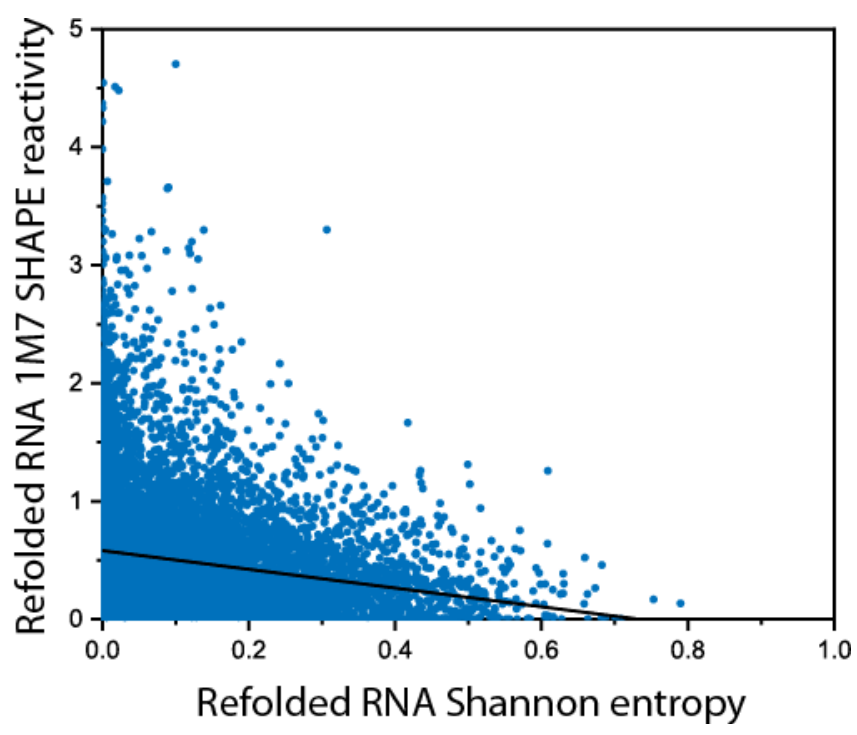

Figure S2: Lack of strong correlation between refolded RNA 1M7 SHAPE reactivities and Shannon entropies. Pearson's $r=-0.19$. Negative 1M7 SHAPE reactivities were set to zero. Pseudoknotted base pairs are ignored, as they have Shannon entropies of zero in our calculations. 


\section{A}
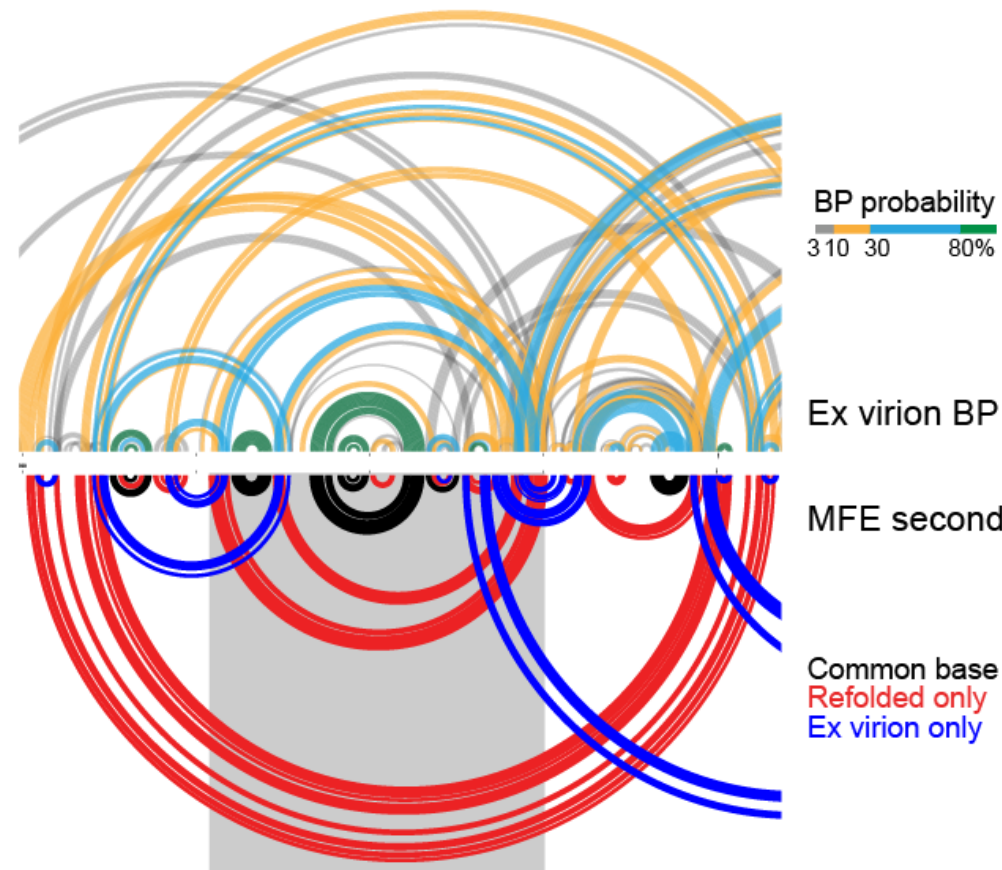

Ex virion BP probabilities

MFE secondary structures

Common base pairs

Refolded only

Ex virion only

Element \#3

B

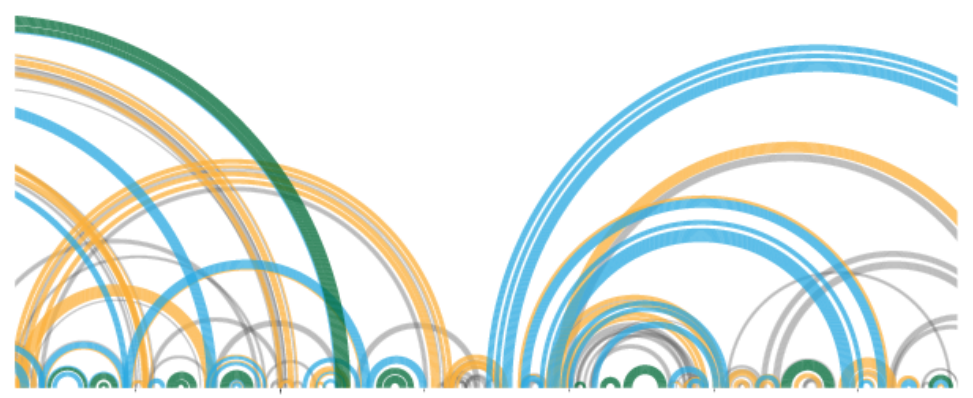

Ex virion BP probabilities

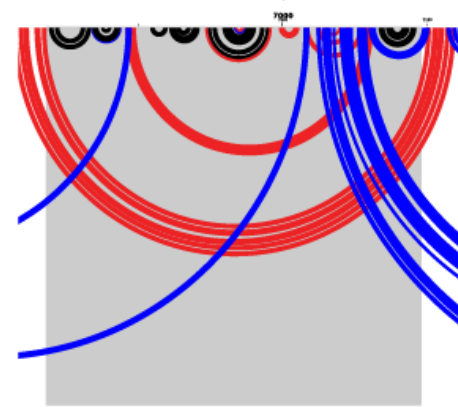

Element \#16

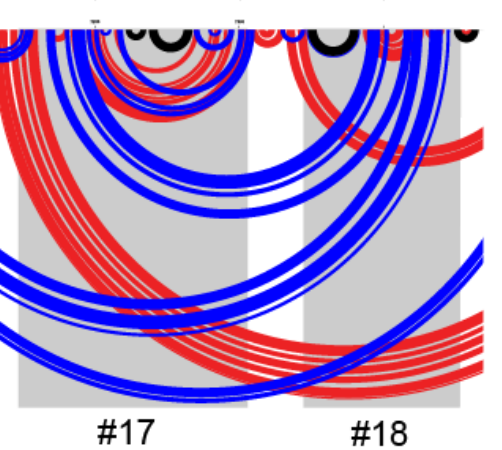

\#17

\#18

Figure S3: Base-pair probability arcs for low sensitivity elements. Ex virion RNA base-pair probability plots and MFE secondary structures for elements $(A) \# 3$ and $(B) \# 16,17$, and 18. (Top) Ex virion base pairs are colored by their probability (see key), with green arcs indicating the most probable helices. (Bottom) MFE secondary structure comparison of refolded and ex virion RNAs. Base pairs common to both RNAs are black, and base pairs unique to the refolded or ex virion models are red and blue, respectively. The gray boxes indicate elements of low SHAPE and low entropy identified in the refolded RNA state. Many of the base-pairs unique to the refolded RNA model (inverted red arcs) are predicted to populate the ex virion base pair ensemble but have low probabilities (gray, yellow, and blue arcs). 
A

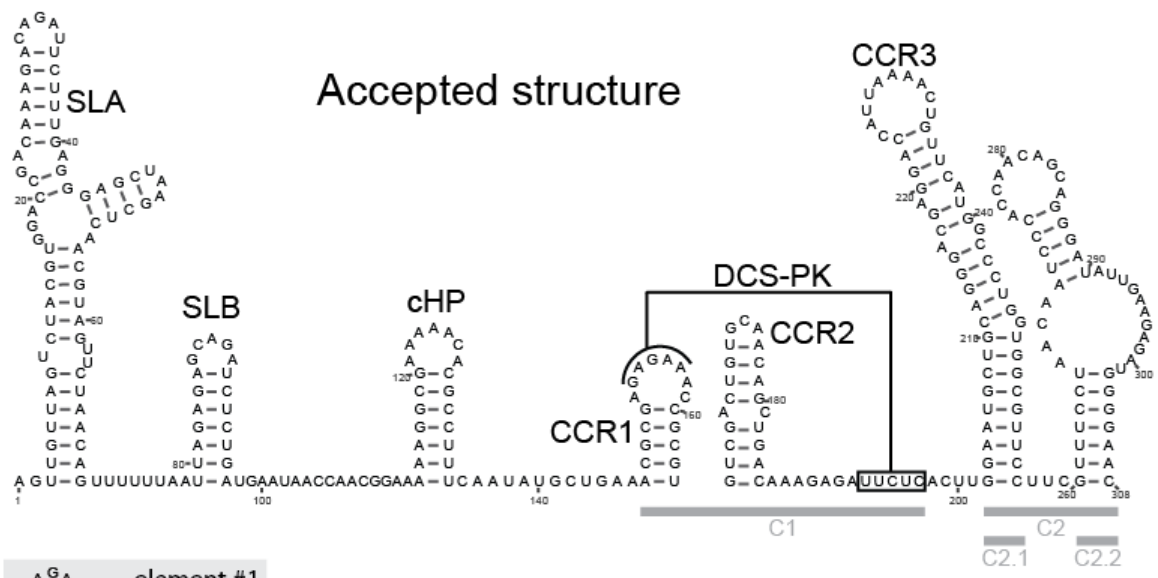

$\mathrm{B}$

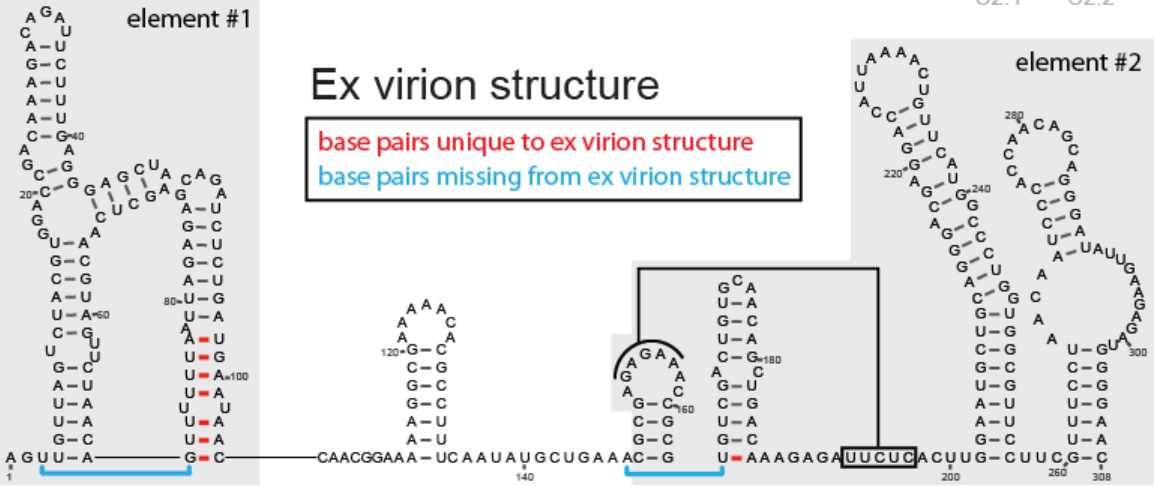

C

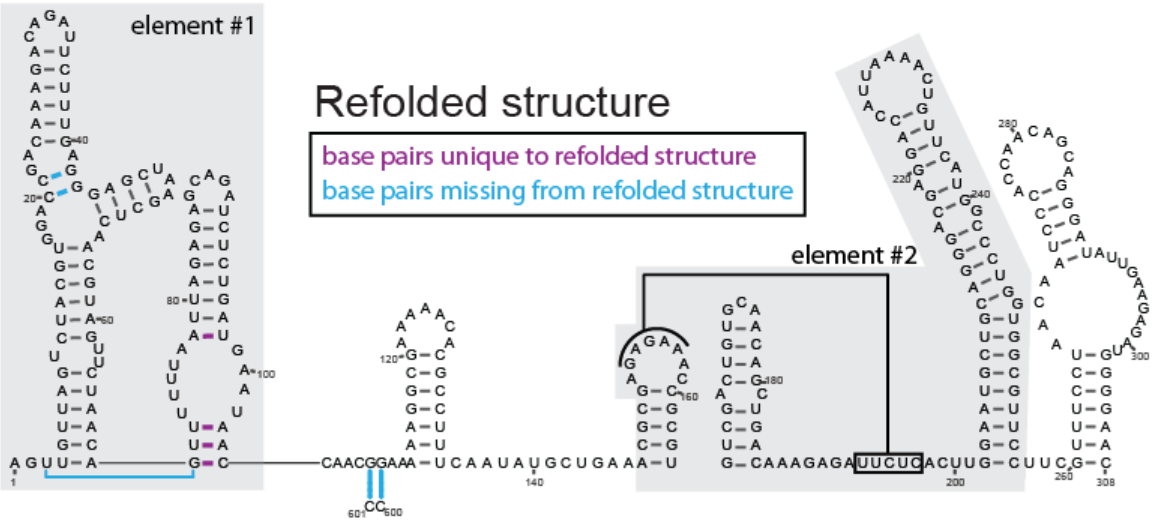

Figure S4: DENV2 RNA secondary structure of the $5^{\prime}-U T R$ and $5^{\prime}$ capsid-coding region. (A) Accepted DENV2 structure, where known structural features (SLA, SLB, CHP, CCR1, CCR2, CCR3, and DCS-PK) are labeled. Labels of features downstream of CHP include naming conventions from Harris et al. in black ${ }^{6}$ and deBorba et al. in gray ${ }^{8}$. (B) Ex virion SHAPE-directed structure model. Base pairs unique to the ex virion RNA model (compared to the accepted structure) are in red, and missing base pairs are colored blue. lowSS structural elements are indicated by gray boxes. (C) Refolded SHAPE-directed structure model. Base pairs unique to the refolded structure (compared to the accepted structure) are in purple, and missing base pairs are colored blue. There are two nucleotides between SLB and cHP (nt 110-111) that are proposed to be base paired to nucleotides 600-601. 


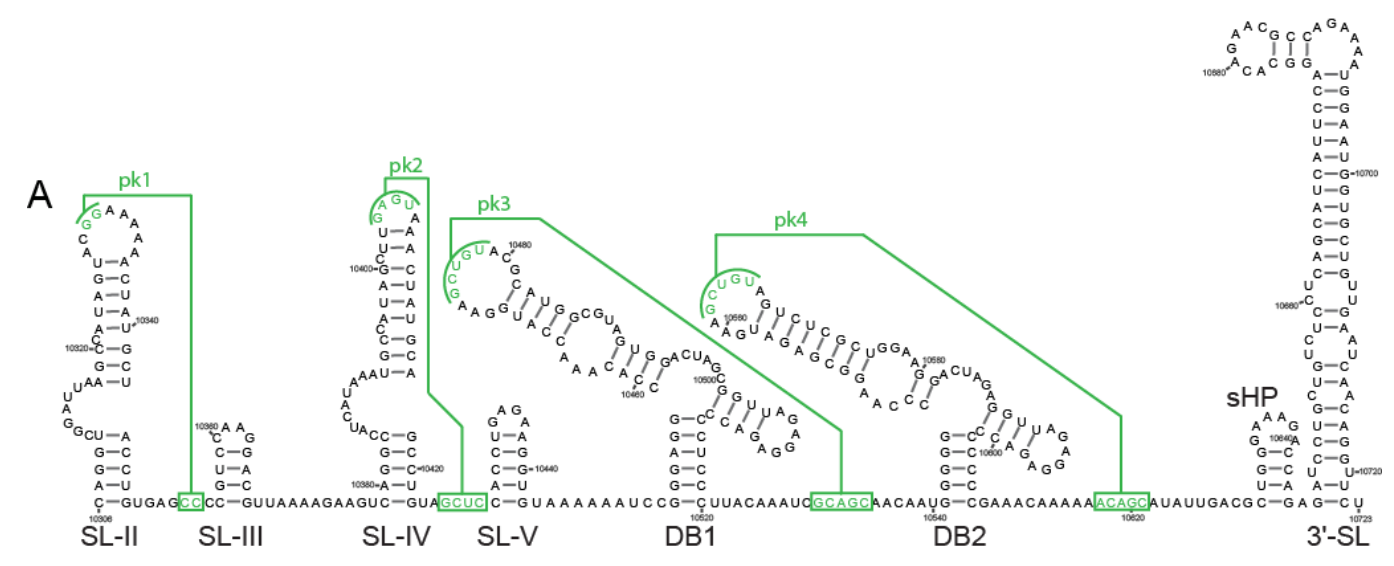

B Accepted structure
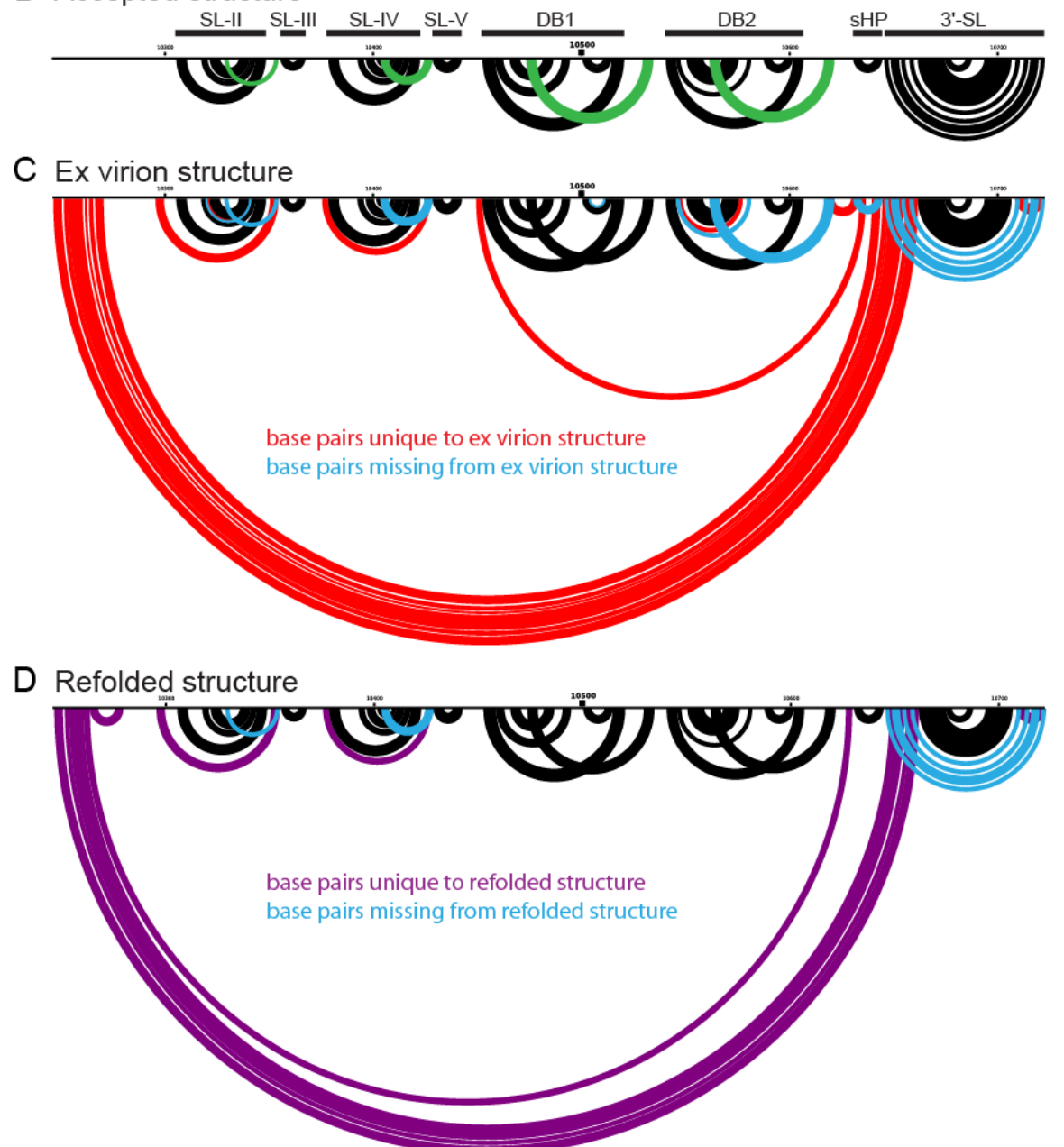

Figure S5: DENV2 RNA secondary structure of the 3'-UTR. (A, B) Accepted DENV2 structure, where known structural features (SL-II, SL-III, SL-IV, SL-V, DB1, DB2, sHP, and 3'SL) are labeled. Pseudoknots are depicted in green. Secondary structures are shown in both (A) conventional and (B) arc plot representations. (C) Ex virion SHAPE-directed structure model. Base pairs unique to the ex virion RNA model (compared to the accepted structure) are in red, and missing base pairs are colored blue. (D) Refolded SHAPE-directed structure model. Base pairs unique to the refolded structure (compared to the accepted structure) are in purple, and missing base pairs are blue. 


\section{Supporting Files:}

SHAPE-derived refolded RNA model (.ct file)

SHAPE-derived ex virion RNA model (.ct file)

Table S1: Refolded SHAPE reactivities

- excel file with 1M7, 1M6, NMIA reactivities

Table S2: Ex virion SHAPE reactivities

- excel file with 1M7, 1M6, NMIA reactivities

Table S3: Refolded RNA structural elements

- includes start/stop sites for all low SHAPE/low Shannon elements

Table S4: Ex virion RNA structural elements

- includes start/stop sites for all low SHAPE/low Shannon elements (this table was also included in Dethoff et al. PNAS 2018) 


\section{References}

(1) Brinton, M. A., and Dispoto, J. H. (1988) Sequence and Secondary Structure-Analysis of the 5'Terminal Region of Flavivirus Genome RNA. Virology 162, 290-299.

(2) Lodeiro, M. F., Filomatori, C. V., and Gamarnik, A. V. (2009) Structural and functional studies of the promoter element for dengue virus RNA replication. J. Virol. 83, 993-1008.

(3) Polacek, C., Foley, J. E., and Harris, E. (2009) Conformational changes in the solution structure of the dengue virus 5 " end in the presence and absence of the 3 " untranslated region. J. Virol. 83, 1161-1166.

(4) Clyde, K., and Harris, E. (2006) RNA secondary structure in the coding region of dengue virus type 2 directs translation start codon selection and is required for viral replication. J. Virol. 80 , 2170-2182.

(5) Liu, Z.-Y., Li, X.-F., Jiang, T., Deng, Y.-Q., Zhao, H., Wang, H.-J., Ye, Q., Zhu, S.-Y., Qiu, Y., Zhou, X., Qin, E.-D., and Qin, C.-F. (2013) Novel cis-acting element within the capsid-coding region enhances flavivirus viral-RNA replication by regulating genome cyclization. J. Virol. 87 , 6804-6818.

(6) Groat-Carmona, A. M., Orozco, S., Friebe, P., Payne, A., Kramer, L., and Harris, E. (2012) A novel coding-region RNA element modulates infectious dengue virus particle production in both mammalian and mosquito cells and regulates viral replication in Aedes aegypti mosquitoes. Virology 432, 511-526.

(7) Sztuba-Solinska, J., Teramoto, T., Rausch, J. W., Shapiro, B. A., Padmanabhan, R., and Le Grice, S. F. J. (2013) Structural complexity of Dengue virus untranslated regions: cis-acting RNA motifs and pseudoknot interactions modulating functionality of the viral genome. Nucleic Acids Res. 41, 5075-5089.

(8) de Borba, L., Villordo, S. M., Iglesias, N. G., Filomatori, C. V., Gebhard, L. G., and Gamarnik, A. V. (2015) Overlapping local and long-range RNA-RNA interactions modulate dengue virus genome cyclization and replication. J. Virol. (Simon, A., Ed.) 89, 3430-3437.

(9) Liu, Z.-Y., Li, X.-F., Jiang, T., Deng, Y.-Q., Ye, Q., Zhao, H., Yu, J.-Y., and Qin, C.-F. (2016) Viral RNA switch mediates the dynamic control of flavivirus replicase recruitment by genome cyclization. Elife 5, 39926.

(10) Huber, R. G., Lim, X. N., Ng, W. C., Sim, A. Y. L., Poh, H. X., Shen, Y., Lim, S. Y., Sundstrom, K. B., Sun, X., Aw, J. G., Too, H. K., Boey, P. H., Wilm, A., Chawla, T., Choy, M. M., Jiang, L., de Sessions, P. F., Loh, X. J., Alonso, S., Hibberd, M., Nagarajan, N., Ooi, E. E., Bond, P. J., Sessions, O. M., and Wan, Y. (2019) Structure mapping of dengue and Zika viruses reveals functional long-range interactions. Nat Commun 10, 1408.

(11) Proutski, V., Gould, E. A., and Holmes, E. C. (1997) Secondary structure of the 3' untranslated region of flaviviruses: similarities and differences. Nucleic Acids Res. 25, 1194-1202.

(12) Zeng, L. L., Falgout, B., and Markoff, L. (1998) Identification of specific nucleotide sequences within the conserved 3 '-SL in the dengue type 2 virus genome required for replication. J. Virol. 72, 7510-7522. 
(13) Pijlman, G. P., Funk, A., Kondratieva, N., Leung, J., Torres, S., van der Aa, L., Liu, W. J., Palmenberg, A. C., Shi, P.-Y., Hall, R. A., and Khromykh, A. A. (2008) A highly structured, nuclease-resistant, noncoding RNA produced by flaviviruses is required for pathogenicity. Cell Host Microbe 4, 579-591.

(14) Olsthoorn, R. C., and Bol, J. F. (2001) Sequence comparison and secondary structure analysis of the $3^{\prime}$ noncoding region of flavivirus genomes reveals multiple pseudoknots. RNA 7 , 1370-1377.

(15) Chapman, E. G., Moon, S. L., Wilusz, J., and Kieft, J. S. (2014) RNA structures that resist degradation by Xrn1 produce a pathogenic Dengue virus RNA. Elife 3, e01892.

(16) Chapman, E. G., Costantino, D. A., Rabe, J. L., Moon, S. L., Wilusz, J., Nix, J. C., and Kieft, J. S. (2014) The Structural Basis of Pathogenic Subgenomic Flavivirus RNA (sfRNA) Production. Science 344, 307-310.

(17) Akiyama, B. M., Laurence, H. M., Massey, A. R., Costantino, D. A., Xie, X., Yang, Y., Shi, P.Y., Nix, J. C., Beckham, J. D., and Kieft, J. S. (2016) Zika virus produces noncoding RNAs using a multi-pseudoknot structure that confounds a cellular exonuclease. Science 354, 1148-1152.

(18) Villordo, S. M., Filomatori, C. V., Sánchez-Vargas, I., Blair, C. D., and Gamarnik, A. V. (2015) Dengue virus RNA structure specialization facilitates host adaptation. PLoS Pathog. (Nagy, P. D., Ed.) $11, \mathrm{e} 1004604$.

(19) Dethoff, E. A., Boerneke, M. A., Gokhale, N. S., Muhire, B. M., Martin, D. P., Sacco, M. T., McFadden, M. J., Weinstein, J. B., Messer, W. B., Horner, S. M., and Weeks, K. M. (2018) Pervasive tertiary structure in the dengue virus RNA genome. Proc. Natl. Acad. Sci. U.S.A. 115, 11513-11518. 